\title{
Ecología de la comunidad de saurios diurnos de la Reserva Nacio- nal de Paracas, Ica, Perú
}

\section{Ecology of the diurnal lizards community in the Reserva Nacional de Pa- racas, Ica, Peru}

\author{
José Pérez Z.1,2,3 y Katya Balta ${ }^{1,3}$
}

\begin{abstract}
${ }^{1}$ Museo de Historia Natural. Universidad Nacional de Mayor de San Marcos. Lima, Perú.

2 Departamento de Ecologia. Instituto de Biologia. Universidade do Estado do Rio de Janeiro (UERJ). Rio de Janeiro, Brasil.

${ }^{3}$ Unidad de Biología de la Conservación. Departamento de Ciencias Biológicas y Fisiológicas. Facultad de Ciencias y Filosofía. Universidad Peruana Cayetano Heredia (UPCH). Lima, Perú.

E-mail, José Pérez Z.: perezz_jm@yahoo.com.br
\end{abstract}

\section{Resumen}

En el presente trabajo estudiamos algunos aspectos de la ecología de la comunidad de saurios diurnos de la Reserva Nacional de Paracas (RNP) desde abril a setiembre de 1999. Analizamos la distribución espacial, el uso del hábitat (nicho espacial), patrones de actividad (nicho temporal) y la dieta (nicho trófico) de estos reptiles, con el objetivo de identificar las principales diferencias que permitirían la división en el uso de los recursos. Estas especies mostraron preferencias por algunos hábitats, a excepción de Microlophus peruvianus, la lagartija de los arenales $M$. theresiae y la lagartija de los gramadales $M$. thoracius icae. Las especies mostraron preferencias por algunos hábitats, a excepción de Microlophus peruvianus que estuvo presente en todos los hábitats. La actividad de los saurios se concentraron al medio día, a excepción de $C$. adspersa. La dieta de estos saurios esta compuesta principalmente por insectos. Los saurios diurnos en la RNP presentan diferencias en el nicho trófico, espacial y/o temporal que posibilitarían su coexistencia en simpatría.

Palabras clave: Saurios, Tropiduridae, uso de recursos, Reserva Nacional de Paracas, Perú.

\section{Abstract}

In present paper we study some ecological aspects of the diurnal saurian community of National Reserve of Paracas (RNP) since April to September 1999.". The spatial distribution, habitat uses (spatial niche), activities patterns (temporal niche) and the diet (trophic niche) of these reptiles were analyzed with the objective of identifying the main differences that will allow a division in the use of the resources. Ctenoblepharis adspersa (bigheaded lizard), Microlophus peruvianus (beach lizard), $M$. theresiae (dune lizard) and $M$. thoracius icae (grass lizard) were the species studied. Each lizard showed preference for some particular habitat, to exception of $M$. peruvianus that was present in all the habitats. The activity was concentrated to the half-day, to exception of $C$. adspersa. The diets were mainly insects. Differences in trophic, temporal and spatial niche were observed and these would facilitate the coexistence in sympatry of species lizards.

Keywords: Saurians, Tropiduridae, use of resources, Reserva Nacional de Paracas, Peru.

\section{Introducción}

La fauna del Desierto Costero Peruano ha sido poco estudiada, a pesar que en el Perú esta ecorregión es una de las más amenazadas por acción del hombre, ya que alberga los mayores centros poblados del país (Rodríguez, 1996). Adicionalmente, es una ecorregión con un considerable número de reptiles endémicos (Carillo e Icochea, 1995). El número de investigaciones realizadas en saurios del Desierto Costero Peruano es escaso, y han sido enfocadas principalmente a la descripción y distribución de especies (Dixon y Wright, 1975; Dixon y Huey, 1970; Schmidt, 1957), y sólo algunas evaluaron aspectos ecológicos (Pérez, 2005b; Catenazi et al., 2005;Pérez y Jhancke, 1998; Werner et al., 1996; Carrillo et al., 1990; Péfaur y López-Tejeda, 1983; Huey, 1979; Huey, 1974).

El Desierto Costero Peruano posee características singulares de extrema aridez y una relativa limitada oferta de alimentos (Brack, 1986), que condicionan a los organismos que habitan este ecosistema a presentar diversas adaptaciones para dividir los recursos y poder coexistir. En los reptiles, estas adaptaciones se presentan generalmente como diferencias en el uso de recursos tróficos, espaciales y/o temporales (Pianka, 1986; Schoener, 1974).

La Reserva Nacional de Paracas (RNP) alberga uno de pocos fragmentos protegidos del Desierto Costero en Perú, y por lo tanto, es el escenario propicio para estudiar las complejas interacciones de la comunidad de saurios en su hábitat natural.

El presente trabajo tiene como objetivo conocer la distribución de los saurios diurnos en la Reserva Nacional de Paracas (RNP), determinar tres aspectos de su nicho (trófico, espacial y temporal), y las estrategias en la división de recursos de las especies de esta comunidad de reptiles.

\section{Área de Estudio}

La RNP (76¹6'15'W, 1351'46”S) se encuentra ubicada en el Departamento de Ica (Fig.1), y forma parte de la ecorregión del Desierto Costero Peruano. La temperatura promedio anual en la $\mathrm{RNP}$ es de $18,7^{\circ} \mathrm{C}$, variando desde $22^{\circ} \mathrm{C}$ en febrero a $15,5^{\circ} \mathrm{C}$ en agosto (INRENA, 2002). La humedad relativa promedio anual es $82 \%$, la precipitación es escasa y proviene principalmente de la condensación de las neblinas invernales (INRENA, 2002). La vegetación en la RNP es reducida, conformada principalmente por vegetación xerofítica como Sessuvium portulocastrum sobre las dunas de arena en algunas playas, Lomas con vegetación marcadamente estacional, y los oasis conformados por parches de vegetación caracterizados por la presencia de grama salada Distichlis spicata, algunos arbustos y el árbol «sofaique» Geoffroea decorticans. 


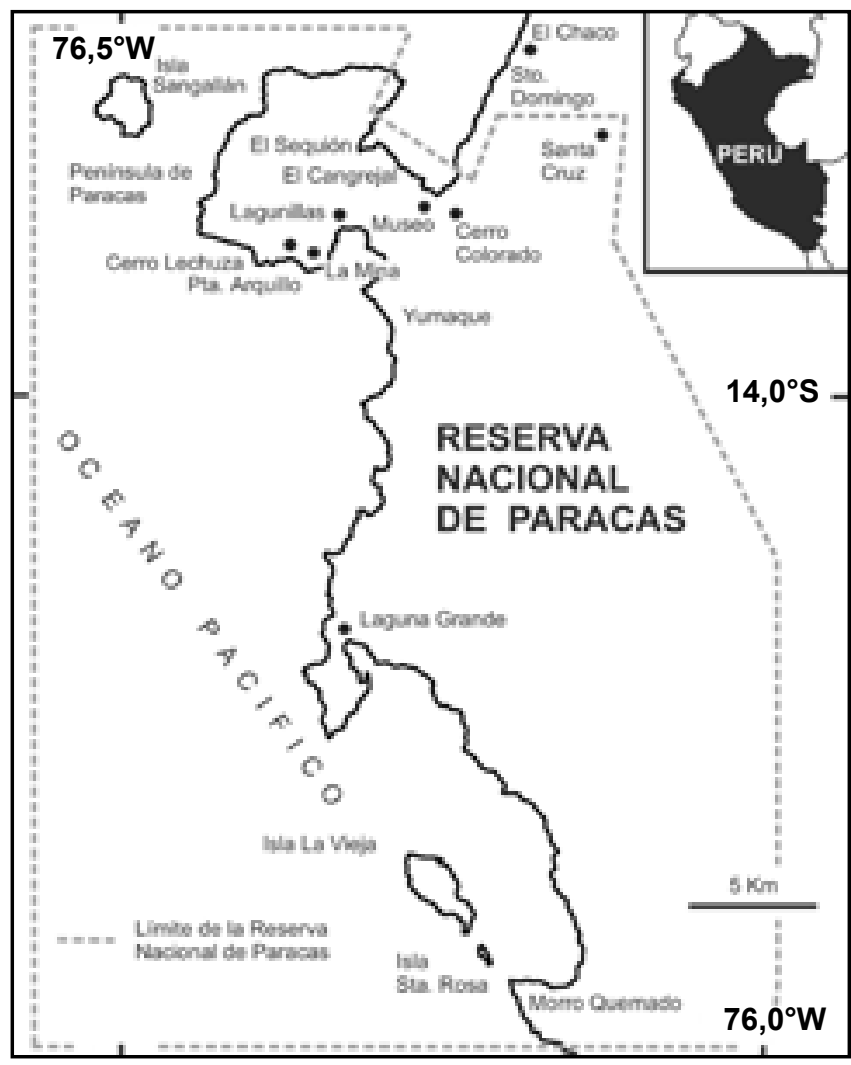

Figura 1. Área de Estudio. Reserva Nacional de Paracas, Ica, Perú, ubicación de las localidades evaluadas

\section{Material y métodos}

Evaluamos la RNP entre abril y septiembre de 1999, meses que corresponden a las estaciones de otoño e invierno. Determinamos cuatro tipos de hábitats de importancia para los saurios: a) Playa: áreas próximas al mar, de arena o piedra, incluyendo dunas de arena con vegetación xerofítica asociada; b) Desierto: sustrato de arena, sin vegetación, con piedras de diferentes tamaños; c) Lomas: sustrato de tierra o arena, con vegetación principalmente arbustiva de porte pequeño, típica de formaciones de Lomas; d) Oasis: sustrato de tierra y salitre con grama salada Distichlis spicata, vegetación arbustiva y arbórea, principalmente el sofaique Geoffroea decorticans.

Las localidades evaluadas incluyen todos los tipos de hábitats mencionados. El hábitat de Playas fue evaluado en las localidades de El Chaco, Santo Domingo, El Cangrejal, Sequión, Punta Arquillo, Lagunillas, La Mina, Yumaque, Laguna Grande, Morro Quemado, y en las islas Sangayán, La Vieja y Santa Rosa (Fig. 1). El hábitat de Desierto fue evaluado en El Chaco, Santo Domingo, Cerro Colorado, Museo, El Cangrejal, Sequión, Punta Arquillo, Lagunillas, La Mina, Península (áreas en el centro de la Península de Paracas), Yumaque, Laguna Grande, Morro Quemado, alrededores de Santa Cruz, y en las islas Sangayán, La Vieja y Santa Rosa. El hábitat de Lomas fue evaluado en Cerro Lechuza, Morro Quemado, y en la isla Sangayán. El hábitat de Oasis fue evaluado en la localidad de Santa Cruz y en el área comprendida entre este punto y la playa de Santo Domingo (Fig. 1).

Las evaluaciones en cada localidad consistieron en transectos de búsqueda cuidadosa en horarios diurnos entre las 8:00—17:00 h, realizados por un grupo de $3-5$ personas. Se realizó un esfuerzo de muestreo semejante en cada uno de los hábitats identificados, para poder realizar comparaciones posteriores entre los hábitats evaluados. Los datos recopilados incluyen información de capturas y registros visuales. Para cada saurio observado registramos datos de la especie, hábitat y microhábitat utilizados por los individuos y hora del registro. A partir de esta información analizamos el nicho espacial y temporal de las especies de saurios. Adicionalmente, para los individuos capturados registramos longitud corporal (SVL) y longitud de cola mediante un calibrador vernier $(0,1 \mathrm{~mm}$ de precisión), masa mediante una balanza de campo Pesola ${ }^{\circledR}(0,1 \mathrm{~g}$ de precisión), sexo y edad (adultos, sub-adultos o juveniles).

En el laboratorio disectamos los individuos colectados, separando el sistema digestivo que fue preservando en alcohol al $70 \%$. Analizamos el contenido estomacal con ayuda de un microscopio estereoscópico, identificando cada item hasta el

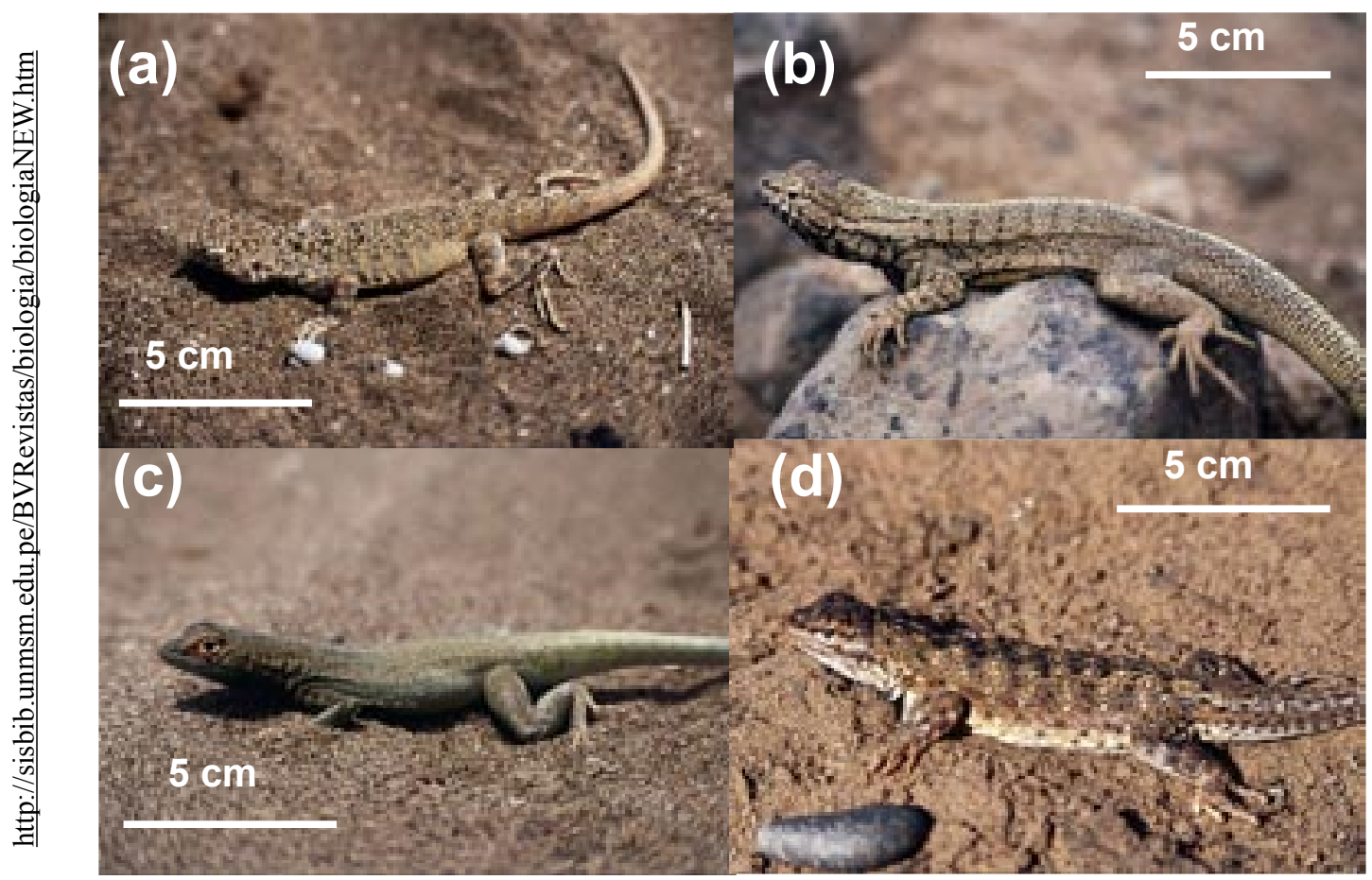

Figura 2. Especies de saurios diurnos en la Reserva Nacional de Paracas, Ica, Perú. Las fotos muestran a individuos machos adultos de: (a) Ctenoblepharis adspersa Tschudi, 1845, (b) Microlophus peruvianus Lesson, 1826, (c) Microlophus theresiae Steindachner, 1902 , y (d) Microlophus thoracicus icae Dixon y Wright, 1975 
nivel de Orden. Agrupamos en la categoría denominada RANI (Restos de Artrópodos No Identificados) a todas las partes de artrópodos que no fue posible identificar. Reunimos todos los restos de plantas encontrados en la categoría Material Vegetal. Registramos el número y frecuencia de cada item alimentario para cada especie de saurio.

Utilizamos el índice de Simpson (Krebs, 1999) para evaluar las amplitudes del nicho (trófico, espacial y temporal) de cada especie, mediante la fórmula:

$$
\mathrm{B}_{i j}=1 / p_{i}^{2}
$$

donde $p_{i}$ es la proporción del recurso $i$. Estimamos las sobreposiciones en el uso de los recursos en estos tres aspectos del nicho mediante la fórmula del coeficiente de sobreposición (Pianka, 1986):

$$
\mathrm{O}_{\mathrm{jk}}=\frac{\Sigma p_{i j} p_{i k}}{\left(\Sigma p_{i j}{ }^{2} \Sigma p_{i k}{ }^{2}\right)^{1 / 2}}
$$

donde $i$ es el tipo de recurso, y $j$ y $k$ son las especies de saurios. Los valores de esta fórmula varían entre 0 (ausencia de sobreposición) y 1 (completa sobreposición). Consideramos una sobreposisicón alta $(1,00-0,60)$, media $(0,55-0,25)$ y baja $(0,20-0,00)$.

\section{Resultados}

Registramos un total de seis especies de saurios en la RNP, cuatro especies de lagartijas de la Familia Tropiduridae: la

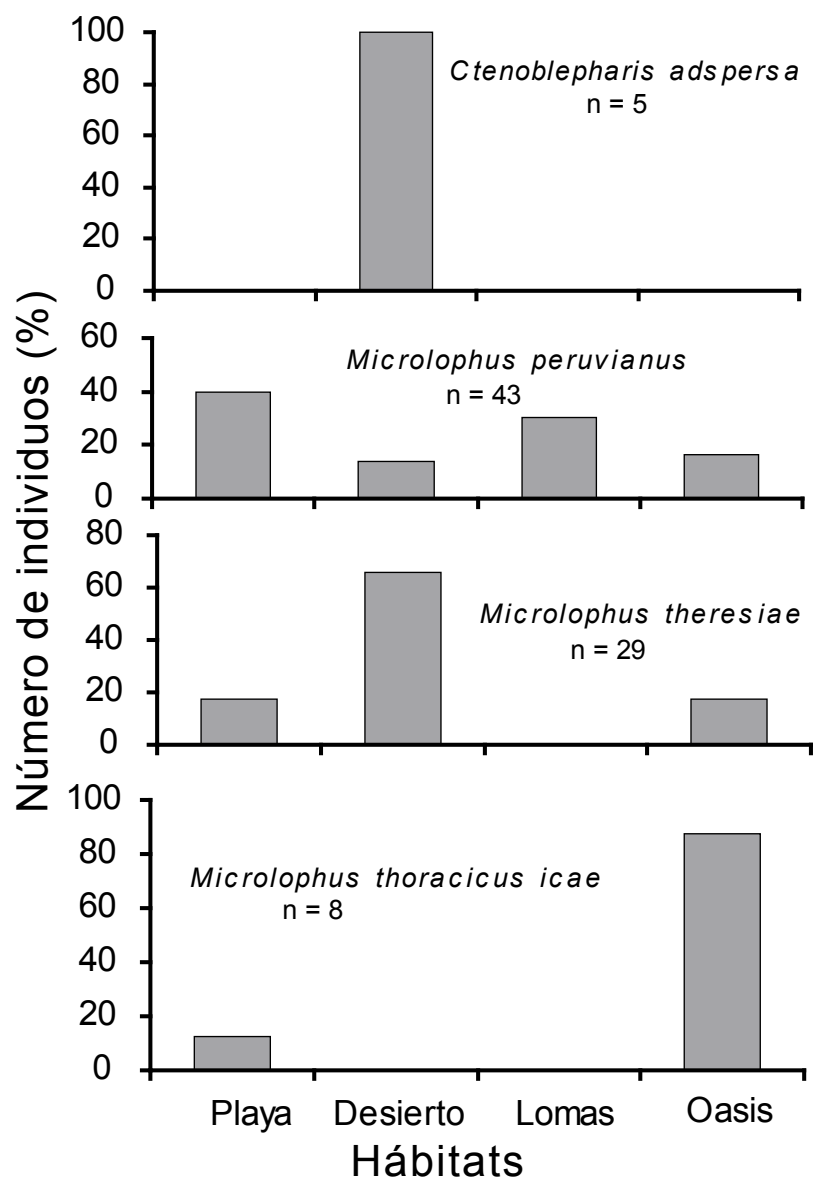

Figura 3. Uso de los hábitats por la especies de saurios diurnos de la Reserva Nacional de Paracas, Ica, Perú. cabezona Ctenoblepharis adspersa Tschudi, 1845, la lagartija de las playas Microlophus (anteriormente Tropidurus (Frost, 1992)) peruvianus Lesson, 1826, la lagartija de los arenales $M$. theresiae Steindachner, 1902 y la lagartija de los gramadales $M$. thoracicus icae Dixon y Wright, 1975 (Fig. 2). Todas estas especies de actividad diurna. Adicionalmente, durante las evaluaciones diurnas registramos individuos inactivos de dos especies de la Familia Gekkonidae, el geco de Paracas Phyllodactylus angustidigitus Dixon y Huey, 1970 y el geco del sur P. gerrhopygus Wiegmann, 1835, ambos de hábitos nocturnos.

\section{Uso de hábitats y distribución en la RNP}

La especie de saurio más abundante fue $M$. peruvianus con cerca de $51 \%$ de los registros. Esta especie también fue la única lagartija presente en todos los hábitats de la RNL (Fig. 3). La Isla Santa Rosa fue la única localidad evaluada donde no registramos ninguna especie de saurio. Ctenoblepharis adspersa $(\mathrm{n}=5)$ fue registrada únicamente en el hábitat de Desierto (Fig. 3), en las localidades de El Chaco, Museo y Morro Quemado (Fig. 4), donde fue registrada en sintopía con juveniles de $M$. peruvianus y de $M$. theresiae.

Los hábitats donde $M$. peruvianus $(\mathrm{n}=43)$ fue más frecuentemente registrada fueron la Playa (39,5\%) y la Loma (30,2\%) (Fig. 3). Microlophus peruvianus estuvo presente en todas las localidades evaluadas, a excepción de la isla Santa Rosa (Fig. 4). Esta lagartija fue la única especie diurna registrada en las islas Sangayán y La Vieja, y en las Lomas evaluadas (Cerro Lechuza, Morro Quemado e isla Sangayán). Microlophus perwianus fue registrada

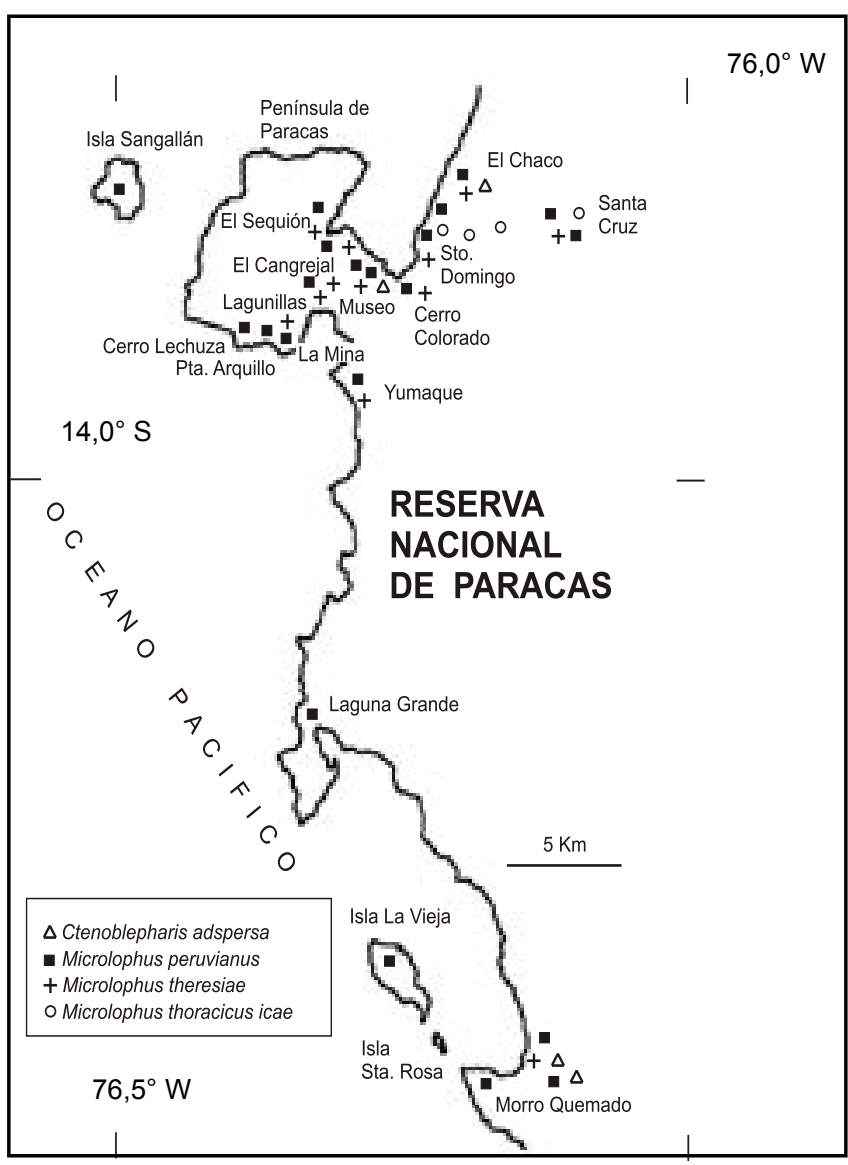

Figura 4. Registro de las especies de saurios diurnos en las diferentes localidades de la Reserva Nacional de Paracas, Ica, Perú. 
Tabla 1. Indices de Amplitud de Nicho $\left(B_{i j}\right)$ y Sobreposición $\left(\mathrm{O}_{\mathrm{jk}}\right)$ en uso de hábitats, actividad y dieta para las especies de saurios diurnos en la Reserva Nacional de Paracas, Ica, Perú. Cteno: Ctenoblepharis adspersa, Peru: Microlophus peruvianus, There: Microlophus theresiae, Thora: Microlophus thoracicus icae.

\begin{tabular}{|c|c|c|c|c|}
\hline $\begin{array}{l}\text { Habitat } \\
\mathrm{O}_{j \mathrm{k}}\end{array}$ & Cteno & Peru & There & Thora \\
\hline Cteno & - & & & \\
\hline Peru & 0,26 & - & & \\
\hline There & 0,94 & 0,50 & - & \\
\hline Thora & 0,00 & 0,40 & 0,28 & - \\
\hline $\mathbf{B}_{\mathrm{ij}}$ & 1,00 & 3,41 & 2,05 & 1,28 \\
\hline $\begin{array}{l}\text { Actividad } \\
\mathrm{O}_{\mathrm{jk}}\end{array}$ & Cteno & Peru & There & Thora \\
\hline Cteno & - & & & \\
\hline Peru & 0,31 & - & & \\
\hline There & 0,44 & 0,69 & - & \\
\hline Thora & $0, .16$ & 0,61 & 0,62 & - \\
\hline $\mathbf{B}_{\mathrm{ij}}$ & 3,57 & 4,96 & 4,57 & 2,33 \\
\hline Dieta & & & & \\
\hline & Cteno & Peru & There & Thora \\
\hline Cteno & - & & & \\
\hline Peru & 0,67 & - & & \\
\hline There & 0,41 & 0,46 & - & \\
\hline
\end{tabular}

en simpatría con las otras tres especies de lagartijas, aunque la sintopía fue registrada sólo entre juveniles de $M$. peruvianus y adultos o juveniles de las otras especies de lagartijas. En el área utilizada por los adultos de $M$. peruvianus no se registraron individuos de otras especies, sin embargo, el área usada por los juveniles de $M$. peruvianus es compartida con juveniles y adultos de $M$. theresiae y/o C. adspersa en algunas localidades como en la playa El cangrejal (Fig. 4).

El principal hábitat para $M$. theresiae $(\mathrm{n}=29)$ fue el Desierto (65,5\%) (Fig. 3) en las localidades de El Chaco, Península, Cerro Colorado y La Mina. Registramos a $M$. theresiae hasta una distancia aproximada de $6 \mathrm{Km}$ del mar en el Oasis Santa Cruz (Fig. 4).

Los registros de Microlophus thoracicus icae $(\mathrm{n}=8)$ estuvieron restringidos a hábitats con presencia de vegetación, como el hábitat de Oasis (87,5\%) (Fig. 3) en Santa Cruz (Fig. 4) y en la playa Santo Domingo (12,5\%). No registramos a $M$. thoracicus icae en hábitat de Lomas.

La amplitud del nicho espacial fue mayor en $M$. peruvianus (3,41), y el menor valor fue registrado para C. adspersa $(1,00)$. La sobreposición en el nicho espacial fue alta entre $C$. adspersa y $M$. theresiae $(0,94)$, fue media para el resto de los pares de especies, excepto entre $C$. adspersa y $M$. thoracicus icae, donde no hubo sobreposición espacial (Tabla 1).

\section{Actividad}

El periodo de actividad de las lagartijas diurnas estuvo restringido entre las 09:00-16:00 h (Fig. 6). Registramos a C. adspersa $(\mathrm{n}=5)$ sólo entre las 09:00 - 11:00 h y al final de la tarde a las 16:00 h (Fig. 5).
El periodo de actividad de $M$. peruvianus $(\mathrm{n}=35)$ fue entre las 9:00 - 15:00 h, presentando un pico de actividad cerca de las 14:00 h (Fig. 5). Registramos a $M$. theresiae $(\mathrm{n}=24)$ en actividad entre 09:00 - 14:00 h, con un mayor número de registros entre 11:00 - 13:00 h. El periodo de actividad de M. thoracicus icae ( $\mathrm{n}=$ 7) estuvo limitado entre 11:00-14:00 h, siendo mas frecuente a las 12:00 h.

La amplitud del nicho temporal fue mayor en $M$. peruvianus $(4,96)$, y el menor valor fue registrado para $M$. thoracicus icae $(2,33)$ (Tabla 1). La sobreposición en el nicho temporal fue alta entre $M$. peruvianus y $M$. theresiae $(0,69), M$. theresiae y $M$. thoracicus icae $(0,62)$, y entre $M$. peruvianus y $M$. thoracicus icae $(0,61)$, fue media para el resto de pares de especies, excepto $C$. adspersa y $M$. thoracicus icae $(0,16)$, donde la sobreposición fue baja (Tabla 1).

\section{Dieta}

No registramos estómagos vacíos en ninguna de las especies. Los ítem más consumidos por los saurios en términos numéricos fueron los dípteros, homópteros y coleópteros (Tabla 2), representando en conjunto el 74,3\% del número de ítem consumidos por la comunidad de saurios. Los coleópteros fueron el ítem más frecuente $(62,1 \%)$ en la dieta general de esta comunidad de saurios, seguidos de los dípteros $(55,2 \%)$ e himenópteros $(41,4 \%)$. A excepción de $C$. adspersa, todas las especies presentaron una dieta caracterizada por un ítem principal (Tabla 2). El consumo de material vegetal fue reducido y registrado sólo en $M$. peruvianus y $M$. thoracicus icae (Tabla 2).

En términos numéricos $C$. adspersa $(\mathrm{n}=4)$ consumió principalmente himenópteros $(29,0 \%)$, coleópteros $(25,8 \%)$ y larvas de insectos $(19,4 \%$ ) (Tabla 2). Los himenópteros fueron los ítem más frecuentes $(75 \%)$.

Los ítem principales en términos numéricos presentes en la dieta de $M$. peruvianus $(\mathrm{n}=10)$ fueron los coleópteros $(54,5 \%)$, seguidos por himenópteros y material vegetal (ambos 10,9\%). Los coleópteros fueron los ítem más frecuentemente registra-
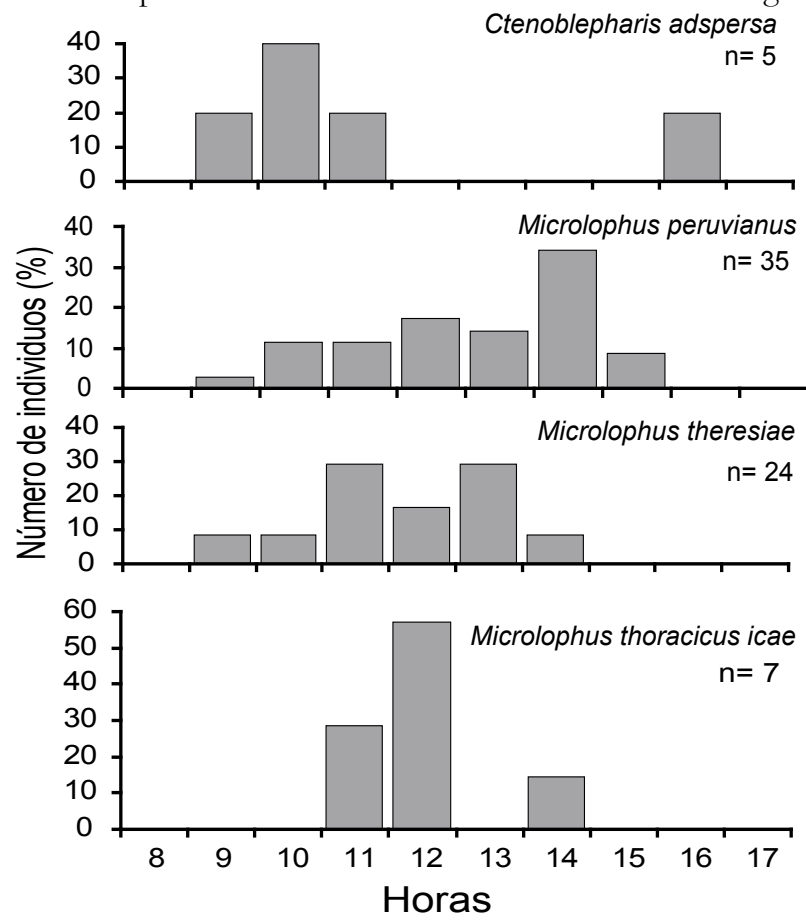

Figura 5. Actividad de las especies de saurios diurnos de la Reserva Nacional de Paracas, Ica, Perú. 
Tabla 2. Dieta de las especies de saurios diurnos en la Reserva Nacional de Paracas, Ica, Perú. Se indican el número $(\mathrm{N})$ y frecuencia $(\mathrm{F})$ para cada categoria de item alimentario con sus respectivos porcentajes. RANI : restos de artrópodos no identificados.

\begin{tabular}{|c|c|c|c|c|c|c|c|c|c|c|c|c|c|c|c|c|}
\hline \multirow[b]{2}{*}{ Item Alimenticio } & \multicolumn{4}{|c|}{$\begin{array}{c}\text { Ctenoblepharis adspersa } \\
\mathrm{n}=4\end{array}$} & \multicolumn{4}{|c|}{$\begin{array}{c}\text { Microlophus peruvianus } \\
\mathrm{n}=10\end{array}$} & \multicolumn{4}{|c|}{$\begin{array}{l}\text { Microlophus theresiae } \\
\qquad \mathrm{n}=9\end{array}$} & \multicolumn{4}{|c|}{$\begin{array}{l}\text { Microlophus thoracicus icae } \\
\qquad \mathrm{n}=6\end{array}$} \\
\hline & $\mathrm{N}$ & $\%$ & $\mathrm{~F}$ & $\%$ & $\mathrm{~N}$ & $\%$ & $\mathrm{~F}$ & $\%$ & $\mathrm{~N}$ & $\%$ & $\mathrm{~F}$ & $\%$ & $\mathrm{~N}$ & $\%$ & $\mathrm{~F}$ & $\%$ \\
\hline Thysanura & - & - & - & - & 2 & 3,6 & 1 & 10,0 & - & - & - & - & - & - & - & - \\
\hline Orthoptera & - & - & - & - & 1 & 1,8 & 1 & 10,0 & 2 & 1,4 & 2 & 22,2 & 1 & 0,8 & 1 & 16,7 \\
\hline Hemiptera & 1 & 3,2 & 1 & 25,0 & - & - & - & - & 4 & 2,8 & 3 & 33,3 & 1 & 0,8 & 1 & 16,7 \\
\hline Homoptera & - & - & - & - & 1 & 1,8 & 1 & 10,0 & 6 & 4,1 & 3 & 33,3 & 79 & 62,2 & 2 & 33,3 \\
\hline Coleoptera & 8 & 25,8 & 1 & 25,0 & 30 & 54,5 & 8 & 80,0 & 26 & 17,9 & 6 & 66,7 & 29 & 22,8 & 3 & 50,0 \\
\hline Lepidoptera & 5 & 16,1 & 2 & 50,0 & - & - & - & - & 12 & 8,3 & 3 & 33,3 & - & - & - & - \\
\hline Diptera & 2 & 6,5 & 2 & 50,0 & 5 & 9,1 & 4 & 40,0 & 78 & 53,8 & 7 & 77,8 & 4 & 3,1 & 3 & 50,0 \\
\hline Hymenoptera & 9 & 29,0 & 3 & 75,0 & 6 & 10,9 & 4 & 40,0 & 5 & 3,4 & 3 & 33,3 & 4 & 3,1 & 2 & 33,3 \\
\hline Larvas Insectos & 6 & 19,4 & 1 & 25,0 & 1 & 1,8 & 1 & 10,0 & 4 & 2,8 & 1 & 11,1 & 2 & 1,6 & 1 & 16,7 \\
\hline Araneae & - & - & - & - & 1 & 1,8 & 1 & 10,0 & 4 & 2,8 & 4 & 44,4 & 1 & 0,8 & 1 & 16,7 \\
\hline Solifuga & - & - & - & - & 1 & 1,8 & 1 & 10,0 & - & - & - & - & 1 & 0,8 & 1 & 16,7 \\
\hline Pseudoescorpionida & - & - & - & - & - & - & - & - & 2 & 1,4 & 2 & 22,2 & 1 & 0,8 & 1 & 16,7 \\
\hline RANI & - & - & - & - & - & - & - & - & 1 & 0,7 & 1 & 11,1 & 2 & 1,6 & 2 & 33,3 \\
\hline Muda de Piel & - & - & - & - & 1 & 1,8 & 1 & 10,0 & 1 & 0,7 & 1 & 11,1 & - & - & - & - \\
\hline Material Vegetal & - & - & - & - & 6 & 10,9 & 5 & 50,0 & - & - & - & - & 2 & 1,6 & 2 & 33,3 \\
\hline TOTAL & 31 & & & & 55 & & & & 145 & & & & 127 & & & \\
\hline
\end{tabular}

$\operatorname{dos}(80 \%)$. Microlophus theresiae $(\mathrm{n}=9)$ consumió principalmente dípteros $(53,8 \%)$ y coleópteros $(17,9 \%)$. Los dípteros fueron los ítem más frecuentes $(78 \%)$.

Los item más consumidos por $M$. thoracicus icae $(\mathrm{n}=6)$ fueron, respectivamente, los homópteros (62,2\%) y coleópteros (22,8\%). Los coleópteros y dípteros fueron los ítem más frecuentes (ambos 50\%).

La amplitud del nicho trófico fue mayor en C. adspersa (4,55), y el menor valor fue registrado en $M$. thoracicus icae $(2,26)$ (Tabla 2). La sobreposición en el nicho trófico fue alta entre $C$. adspersa y $M$. peruvianus $(0,67)$, fue media para el resto de pares de especies, excepto entre $M$. theresiae y $M$. thoracicus icae $(0,22)$ donde registramos una baja sobreposición (Tabla 1).

\section{Discusión}

El presente trabajo brinda los primeros datos cuantitativos sobre algunos aspectos ecológicos de las especies de saurios del Desierto Costero Peruano. Como producto de esta investigación proporcionamos una lista completa y distribución de los saurios presentes en la RNP, además de aportar los primeros datos sobre uso de hábitats, patrones de actividad, dieta y división de los recursos de una comunidad de saurios costeros en Perú.

Evidenciamos una aparente restricción (mínima amplitud de nicho espacial) de C. adspersa al hábitat de Desierto, y un menor número de registros comparado con las otras especies evaluadas, estas dos características también fueron observadas en poblaciones de la Reserva Nacional de Lachay (datos no publicados), y motivaron la inclusión de $C$. adspersa como especie Casi Amenazada por Icochea (1998) y Vulnerable por INRENA (2004), según las categorías de la UICN (2001). Esta es la única especie de saurio presente en la RNP incluida en alguna categoría de amenaza (INRENA, 2004). La restricción en el tipo de hábitat que presenta $C$. adspersa contrasta con la amplitud en el nicho trófico, el mayor registrado entre las especies evaluadas, a pesar del reducido número de individuos analizados.
La lagartija de las playas $M$. peruvianus es la especie más conspicua del Desierto Costero Peruano (Dixon y Wrigth, 1975), fue la especie más frecuente en los registros en nuestro estudio, y estuvo presente en todos los tipos de hábitats (mayor amplitud en el nicho espacial). Registramos a $M$. peruvianus en sus hábitats característicos como playas, Desierto y Oasis, así como también en hábitats de Lomas. En otras Lomas del Desierto Costero, ha sido reportado $M$. tigris Tschudi, 1845 (Pérez, 2005b; Dixon y Wrigth, 1975), saurio que no hemos registrado en la RNP. Este reemplazo de especies en las Lomas, se debería a una menor humedad en las Lomas de la RNP, lo que se ve reflejado en el menor grado de complejidad de la vegetación que presentan las Lomas de la RNP. Las Lomas evaluadas en la RNP (Cerro Lechuza, Morro Quemado e isla Sangayán) no presentan árboles ni arbustos de porte medio. Aparentemente, esta baja humedad posibilitaría que $M$. peruvianus habite estas Lomas, y no sean ocupadas por $M$. tigris. Microlophus peruvianus es una de las especies del género de mayor tamaño en Perú (Dixon y Wright, 1975), posee un comportamiento fuertemente territorial (Péfaur y LópezTejeda, 1978). Estas características proporcionarían una ventaja competitiva a esta especie, que se reflejaría en la exclusividad sobre las áreas que ocupan los adultos de $M$. peruvianus.

Microlophus theresiae anteriormente había sido reportado sólo en el hábitat de Playas, alejados del mar a una distancia máxima de $500 \mathrm{~m}$ (Dixon y Wright, 1975). Nosotros registramos a esta especie, principalmente en el hábitat de Desierto y también en el Oasis de Santa Cruz, a varios kilómetros del mar, evidenciando una menor dependencia del hábitat de Playa, y el uso de un mayor número de tipos de hábitats de los reportados anteriormente. Por otro lado, como Dixon y Wright (1975) reportaron, observamos una diferencia entre la preferencia de los hábitats utilizados entre juveniles y adultos. Los juveniles son frecuentes en playas de sustrato de arena y los adultos en acantilados de rocas, sin embargo, cabe destacar que si las playas presentan numerosos lugares adecuados para refugio y alimentación (rocas 
o vegetación xerofítica), estas serían ocupadas preferentemente por M. peruvianus adultos. Las playas con aparente menor oferta de refugios y alimento son ocupadas por $M$. theresiae. Tanto juveniles como adultos de $M$. theresiae están presentes en el hábitat de Desierto y Oasis.

La presencia de juveniles de $M$. thoracicus icae en la playa Santo Domingo ha sido observada anteriormente (datos no publicados). La subespecie $M$. thoracicus icae tiene una amplia distribución a lo largo del valle del río Ica (Dixon y Wrigth, 1975), al este de la RNP, y llegaría en algunas localidades hasta las inmediaciones del mar (como por ejemplo la playa Santo Domingo) mediante el uso de la vegetación que conforman el hábitat de Oasis, que se ubican entre el valle del río Ica y las playas. Este corredor natural estaría posibilitando la distribución de $M$. thoracicus icae. Por otro lado, cabe mencionar que no observamos este efecto de corredor natural en el caso del geco de las Lomas Phyllodactylus lepidopygus Tschudi, 1845. Este geco también está presente en el valle del río Ica (Dixon y Huey, 1970), pero no ha sido registrado en la RNP. Phyllodactylus lepidopygus aparentemente no podría utilizar la vegetación de los oasis, porque no presentarían el tipo de hábitat adecuado para este geco.

La sobreposición en el uso de los hábitats registrada entre las especies evaluadas fue menor en $M$. thoracicus icae, debido a que este saurio esta prácticamente restringido al hábitat de Oasis, y no ocuparía frecuentemente los otros habitats de la RNP. Por lo tanto, $M$. thoracicus icae no estaría presente en el Desierto, uno de los hábitats más usados por la comunidad de lagartijas diurnas en la RNP. Las especies con mayor sobreposición en el uso de los hábitats son $C$. adspersa y $M$. theresiae, debido a que ambas utilizan el Desierto como su hábitat principal.

Microlophus peruvianus, $M$. theresiae y $M$. thoracicus icae presentan una actividad diaria semejante al unimodal, con un mayor número de registros durante medio día. Esta actividad es frecuente en especies de lagartijas del desierto (Pianka, 1986), y ha sido reportada anteriormente para M. perwianus (Huey, 1974) en el Desierto Costero norte de Perú. Los registros de actividad de $C$. adspersa son reducidos, pero podrían estar indicando un patrón diferente, con picos de actividad al inicio de la mañana y final de la tarde. Si bien el número de registros para esta especie es reducido para realizar una afirmación categórica, este tipo de patrón de actividad en $C$. adspersa ha sido observado anteriormente en otra localidad (datos no publicados). Este horario en la actividad de C. adspersa podría estar reduciendo la sobreposición en el nicho temporal en la comunidad de saurios diurnos en la RNP, y dividiendo las horas de luz solar entre estas especies. Este tipo de estrategia ha sido reportado en otros ecosistemas, como un importante mecanismo de división de recursos en comunidades de saurios (Pianka, 1986).

La amplitud del nicho temporal fue mayor para $M$. peruvianus. Huey (1974) también reporta un periodo de actividad relativamente amplio para $M$. peruvianus en el norte de Perú. Este comparativamente amplio tiempo de actividad (mayor amplitud en el nicho temporal), supondría una ventaja competitiva con las otras especies de saurios diurnos, que sumada a su mayor tamaño, y su presencia en todos los hábitats evaluados, ayudaría a explicar porque $M$. peruvianus es la especie de saurio dominante en la RNP. El amplio periodo de actividad, también implica que sus actividades se realizan en un amplio rango de temperaturas, para lo cual $M$. peruvianus debe tener complejas estrategias de comportamiento y/o fisiológicas para mantener una temperatura corporal adecuada.

Es importante destacar que el período de esta evaluación incluye las estaciones de otoño e invierno, y por tanto el tiempo de actividad de los saurios fueron las menores del año. Evaluaciones posteriores de estas mismas especies, en época de verano con mayores temperaturas ambientales, podrían evidenciar algunos cambios en los patrones de actividad registrados en nuestro estudio.

Cabe mencionar que, también observamos una división temporal diurno/nocturno entre lagartijas y gecos de la RNP. Las lagartijas, presentaron patrones de actividad estrictamente diurnos, sin ningún registro crepuscular o nocturno, y por otro lado no registramos ningún geco activo en horario diurno. En otras localidades se ha reportado actividad crepuscular en algunos individuos del geco Phyllodactylus microphyllus Cope, 1876 (Werner et al., 1996).

A pesar de las limitaciones que representan el reducido tamaño de muestra, contamos con datos que nos permiten establecer algunas características de la dieta de la comunidad de saurios diurnos de la RNP.

La considerable amplitud en los nichos tróficos de las especies evaluadas, sugieren una dieta de tipo generalista, característica de especies de saurios presentes en hábitats con baja oferta de alimento (Dunham, 1983), y en particular de especies de la familia Tropiduridae. Esta amplitud de nicho tendería a disminuir con el incremento de la abundancia de los recursos alimentarios. El carácter generalista de la dieta de los saurios del Desierto Costero Peruano, sólo había sido documentado para $M$. peruvianus (Pérez y Jhancke, 1998; Péfaur y López-Tejeda, 1978), y para M. tigris (Pérez, 2005b). La gran variedad de ítem consumidos por algunas especies del género Microlophus en Perú ha sido evidenciada con registros de canibalismo para $M$. peruvianius (Pérez, 2005a) y $M$. quadrivittatus (Pérez y Balta, 2005b), y saurofagia para $M$. theresiae (Pérez y Balta, 2005a).

La considerable amplitud del nicho trófico de estos saurios, con dietas de tipo generalista y oportunista, explicarían parcialmente la considerable sobreposición en la dieta registrada entre las especies. Por otro lado, las estrechas relaciones filogenéticas entre estas especies de la misma familia y/o género, estarían contribuyendo al incremento de las similaridades en sus dietas, reflejando a su vez estrategias de forrajeo semejantes.

La dieta de C. adspersa, a diferencia de los otros saurios diurnos de la RNP, no presenta un tipo de ítem que destaque claramente, hecho que se refleja en la comparativa mayor amplitud en el nicho trófico registrada para este saurio en esta evaluación. Sin embargo, debido al reducido número de estómagos analizados no podemos llegar a una afirmación concluyente. Los datos aquí presentados constituyen la primera información sobre la dieta de esta especie.

El registro de material vegetal en los estómagos de $M$. peruvianus y $M$. thoracicus icae reflejaría el tipo de dieta omnívoro de estas dos especies. La importancia del material vegetal en la dieta de estas especies ha sido observada en otras localidades del Desierto Costero Peruano (Péfaur y López-Tejeda, 1983; Dixon y Wrigth, 1975). Este hecho explica, por ejemplo, la estrecha relación entre $M$. thoracicus icae con los hábitats con vegetación, 
que sería más evidente en individuos adultos, donde el material vegetal es un ítem de mayor importancia, en comparación con la dieta de juveniles (Dixon y Wrigth, 1975). A pesar que los datos de nuestra evaluación están basados principalmente en individuos juveniles, se evidenció material vegetal en la dieta de $M$. thoracicus icae. El mayor consumo de material vegetal por parte de lagartijas adultas, comparado con juveniles, ha sido reportado para varias especies (Copper y Vitt, 2002; Rocha, 1989; Van Sluys, 1993; Pough, 1973).

Pough (1973) señaló que el consumo de material vegetal en lagartijas es restringido a especies relativamente grandes, peso mayor de $100 \mathrm{~g}$, y sugirió que las especies más pequeñas no pueden digerir tejidos vegetales debido a limitantes fisiológicas y/o morfológicas. Sin embargo, $M$. thoracicus icae es una lagartija relativamente pequeña, con peso promedio aproximado de 15 g, por lo tanto, el consumo de material vegetal sería mucho más difundido de lo presumido entre las lagartijas, como ha sido reportado en otras especies de tropiduridos (ej.: Van Sluys, 1993; Rocha, 1989). El tipo de dieta omnívoro que presenta $M$. peruvianus (Péfaur y López-Tejeda, 1978) y M. thoracicus icae (Dixon y Wrigth, 1975), no fue registrado para $M$. theresiae y C. adspersa.

Según Dixon y Wright, (1975), la dieta de M. theresiae se caracteriza por el consumo de alimentos relacionados al mar como los hemípteros acuáticos. Esta característica en la dieta de $M$. theresiae no fue evidenciada en nuestra evaluación, donde registramos una dieta basada principalmente en ítem de ambientes terrestre (ej.: dípteros, coleópteros, lepidópteros). Los datos aquí presentados constituyen la primera información cuantitativa sobre la dieta de esta especie endémica para Perú.

Las estrategias para la división de recursos en las comunidades de saurios están basadas principalmente en diferencias en los nichos trófico, espacial y/o temporal (Pianka 1986; Schoener, 1977). En la comunidad de saurios diurnos de la RNP registramos diferencias en estos tres aspectos de los nichos. La relativa alta sobreposición en la dieta de algunas parejas de especies de saurios diurnos en la RNP, contrasta con la media o baja sobreposición entre esas mismas especies en el uso de hábitat (ej: $C$. adspersa y $M$. peruvianus presentan una alta sobreposición en el nicho trófico, y una baja sobreposición en el nicho espacial y temporal). No registramos una alta sobreposición en dos aspectos del nicho para ningún par de especies, hecho que podría estar reflejando un tipo de estrategia para evitar una alta sobreposición en los nichos de estas lagartijas. Esta complementariedad en los nichos también ha sido reportada en evaluaciones de otras comunidades de saurios (Pérez, 2005b; Pianka, 1986; Schoener, 1974). Adicionalmente, la aparente división en el nicho temporal, básicamente atribuida al diferente patrón de actividad registrado para C. adspersa, incrementaría las diferencias en el uso de los recursos por esta comunidad. En algunos casos, los depredadores simpátricos presentan diferentes patrones de actividad durante el día (Schoener, 1974). Estas sutiles diferencias en los patrones de actividad, si bien no producen una menor sobreposición en dieta (Huey y Pianka, 1983; Jasick, Greene y Yañez, 1981), permitirían mantener diferencias en otros aspectos, como por ejemplo preferencias termales. Por otro lado, estas diferencias también pueden ser explicadas como estrategias para reducir el riesgo de depredación (Dunham, 1983).

Las características de aridez y relativa escasez de recursos alimentarios que presenta la RNP, condicionan que la comunidad de saurios diurnos que la habitan presenten diferencias en algunas características de su nicho (trófico, espacial y temporal) para reducir la sobreposición en el uso de los recursos y permitan su coexistencia en simpatría.

\section{Agradecimientos}

Agradecemos a Carlos Frederico Duarte Rocha, Mara Cíntia Kiefer, Andreas Schlueter y Davor Vrcibradic por los aportes a este artículo. A Omar Pesantes por su constante apoyo durante todas las etapas de esta investigación. A Eliana Quispitúpac, Alfredo Portilla, Emilio Fuentes, Alberto Guanilo y Jean Mattos por la ayuda en el trabajo de campo. Al personal de la Reserva Nacional de Paracas por el apoyo logístico, en especial a Luis Paz Soldán, Víctor Quispe y Juan Heaton. A Norberta Martínez y Eliana Quispitúpac por la colaboración en la identificación de los invertebrados, a Ernesto Pérez por la diagramación de los gráficos. A Raúl Sanchez y Cecilia Rivas del Grupo de Estudios Ambientales del Perú (GEA-Perú) y a World Wildlife Fund - Oficina Programa Perú (WWF-OPP) por el financiamiento de este proyecto.

\section{Literatura Citada}

Brack, A. J. 1986. Ecología de un País Complejo. En: Gran Geografía del Perú. Naturaleza y Hombre. Vol. II. Editorial ManferMejía Baca, España.

Cano, A. \& K. Young. 1998. Los Pantanos de Villa. Biología y Conservación. Serie de Divulgación $N^{\circ} 11$ del Museo de Historia Natural de la Universidad Nacional Mayor de San Marcos.

Carrillo, N., D. Rothenstein, A. Salas \& Y. Werner. 1990. Radiation an convergence among desert geckos: Phyllodactylus species. resembling both Ptyodactylus and Stenodactylus. Amphibia-Reptilia (11): 1-13.

Catenazi, A; Carrillo, J \& M. Donnelly. 2005. Seasonal and Geographic Eurythermy in a Coastal Peruvian Lizard. Copeia 4: 713-723.

Cooper, W. E. \& L. J. Vitt. 2002. Distribution, extent, and evolution of plant consumption by lizards. Journal Zoological of London 257: 487-517.

Dixon, J. \& R. Huey. 1970. Systematic of the lizards of the gekkonidae genus Phyllodactylus of mainland South America. Contribution in Science The Natural History Museum of Los Angeles. 192: 1-78.

Dixon, J \& J. Wright. 1975. A review of the lizards of the iguanid genus Tropidurus in Peru. Contribution in Science The Natural History Museum of Los Angeles. 1-40.

Dunham, A. E. 1983. Realized niche overlap, resources abundance, and intensity of interspecific competition. En: Huey, R., E. Pianka \& T. Schonener. 1983. Lizard Ecology: studies of a model organism. Harvard University Press. Pp. 261-280.

Frost, D.R. 1992. Phylogenetic analysis and taxonomy of the Tropidurus group of lizards (Iguania:Tropiduridae). American Museum Novitates 3033:1-68.

Huey, R. 1974. Winter thermal ecology of the iguanid lizard Tropidurus peruvianus. Copeia (1):149-155.

Huey, R. 1979. Parapatry and niche complementarity of Peruvian desert geckos (Phyllodactylus): the ambiguous role of competition. Oecologia 38: 249-259.

Huey, R., E. Pianka \& T. Schonener. 1983. Lizard Ecology: studies of a model organism. Harvard University Press.

Icochea, J. 1998. Lista roja preliminar de los anfibios y reptiles amenazados del Departamento de Lima. En: Los Pantanos de Villa. Biología y Conservación. Editores: A. Cano \& K. 
Young. Serie de Divulgación $\mathrm{N}^{\circ} 11$ del Museo de Historia Natural de la Universidad Nacional Mayor de San Marcos. Pp. 217-229.

Jaksic, F. M., H. W. Greene \& J. L. Yañez. 1981. the guild structure of a community of predatory vertebrates in central Chile. Oecologia (Berl.) 49: 21-28.

INRENA (Instituto Nacional de Recursos Naturales). 2002 Reserva Nacional de Paracas. Plan Maestro 2003-2007. Pisco: INRENA. RJ 4652002 INRENA. 192 p.

INRENA. 2004. Lista de especies de fauna silvestre amenazadas de extinción. http://www.inrena.gob.pe/iffs/biodiv/catego_fauna_amenazada.pdf Acceso 30/06/06.

Krebs, C. 1999. Ecological Methodology. Addison Wesley Longman.

Pefaur, J \& E. López-Tejeda. 1983. Ecological notes on the lizard Tropidurus peruvianus in southern Peru. Journal of Arid Environments. 6:155-160.

Pérez Z., J. 2005a. Microlophus peruvianus. Cannibalism. Herpetological Review 36 (1): 63.

Pérez Z., J. 2005b. Ecologia de Duas Espécies de Lagartos Simpatricos em uma Formação Vegetal de Lomas no Deserto
Costeiro Peruano Central. Dissertação de Mestrado. Universidade do Estado do Rio de Janeiro (UERJ). Rio de Janeiro. Brasil.

Pérez Z., J. \& K. Balta. 2005a. Microlophus theresiae. Saurophagy. Herpetological Review. 36 (1): 63.

Pérez Z., J. \& K. Balta. 2005b. Microlophus quadrivittatus. Cannibalism. Herpetological Review. 36 (2): 180.

Pérez, J. \& J. Jhancke. 1998. Saurios como consumidores de ectoparásitos de aves guaneras. Boletín del Instituto del Mar de Perú. 81-86.

Pianka, E. 1986. Ecology and Natural History of Desert Lizards. Princeton University Press. New Jersey.

Pianka, E. 1988. Evolutinary Ecology. Harper \& Row. New York.

Pough, F. A. 1973. The lizard energetics and diet. Ecology 54: 588-596.

Rocha, C. F. D. 1989. Diet of a tropical lizard (Liolaemus lutzae) of southeastern Brazil. Journal of Herpetology 23: 292-294. 